\title{
Prior Immunosuppressive Therapy and Severe IIIness Among Patients Diagnosed with SARS-CoV-2: a Community-Based Study
}

\author{
Fernando S. Velayos, M.D. M.P.H. ${ }^{7}$, Jennifer R. Dusendang, M.P. $H^{2}$, and \\ Julie A. Schmittdiel, Ph.D., M.A. ${ }^{2}$ \\ 'Division of Gastroenterology and Hepatology, Kaiser Permanente San Francisco Medical Center, San Francisco, CA, USA; ${ }^{2}$ Kaiser Permanente \\ Northern California Division of Research, Oakland, CA, USA.
}

BACKGROUND: An estimated 10 million people in the USA are immunocompromised, a risk factor for severe COVID-19. Data informing whether immune-mediated medications lead to more severe infection are sparse.

OBJECTIVE: Determine whether outpatient immunosuppressive therapies that treat autoimmune inflammatory disease or prevent solid organ transplant rejection are associated with severe illness after diagnosis with SARS-CoV-2

DESIGN: Retrospective cohort study

PARTICIPANTS: Adults with a positive PCR nasal swab for SARS-CoV-2 from February 25 to September 9, 2020, cared for within a large integrated health care organization

MAIN MEASURES: Exposure was defined as an outpatient fill of prednisone, immunomodulator, small-molecule, or biologic therapy in the 105 days prior to a positive SARS-CoV-2 PCR test. The main outcome was either hospitalization, ICU admission, or death within 45 days after diagnosis of SARS-CoV-2. Multivariable logistic regression models were adjusted for age, race, gender, body mass index, comorbidities, and autoimmune disease.

KEY RESULTS: A total of 39,686 adults had a positive PCR test. In the primary analysis, prior prednisone use was associated with severe illness after diagnosis with SARS-CoV-2 (odds ratio (OR) 1.31; 95\% confidence interval (CI) 1.081.60); however, immunomodulator (OR 0.88; 95\% CI 0.571.34) and biologic/small-molecule therapy (OR 1.26; 95\% CI 0.79-2.00) were not. Secondary analyses showed variable risk among therapies: Janus-kinase inhibitors had an increased odds of severe illness (OR 3.35; 95\% CI 1.16-9.67), thiopurines/conventionaldisease-modifying antirheumatic drugs had a reduced odds (OR 0.53; 95\% CI 0.32-0.88), and tumor necrosis factor inhibitors were not associated (OR 0.45; 95\% CI 0.18-1.08).

CONCLUSIONS AND RELEVANCE: Outpatient use of prednisone is associated with severe illness after diagnosis of SARS-CoV-2. Immunomodulator and biologic/ small-molecule therapy were not associated, but different risk subgroups were identified. Our findings can inform risk-benefit discussions in the clinic and risk-based recommendations for patients on these therapies.

Received March 12, 2021

Accepted September 10, 2021

Published online September 28, 2021
J Gen Intern Med 36(12):3794-801

DOI: $10.1007 / \mathrm{s} 11606-021-07152-2$

(C) Society of General Internal Medicine 2021

\section{INTRODUCTION}

An estimated 10 million people in the USA have an altered immune system, a potential risk factor for severe and fatal infection from severe acute respiratory syndrome coronavirus2 (SARS-CoV-2). ${ }^{1}$ Patients on immune-modifying therapies such as prednisone, immunomodulators, biologics, and targeted small-molecule therapy are concerned that these medications may unacceptably impair the immune system and allow SARS-CoV-2 to spread unchecked in the body. ${ }^{2-4}$

Despite these concerns, it is unclear whether these immunemodifying therapies, used to treat conditions such as Crohn's disease, ulcerative colitis, rheumatoid arthritis, ankylosing spondylitis, psoriasis, psoriatic arthritis, and systemic lupus erythematosus as well as prevent rejection from solid organ transplantation, in fact worsen the severity of SARS-CoV-2 if infection occurs. ${ }^{5}$ Prior studies have studied highly selected populations from single-specialty international registries or from referral centers, raising questions of generalizability as well as complete capture of key medication exposure, confounders, and outcomes. ${ }^{6-18}$

To address this gap, we assessed the relationship between use of prednisone, immunomodulators, biologics, and targeted smallmolecule therapy prior to the diagnosis of SARS-CoV-2 and subsequent hospitalization, ICU admission, or death, all within a single integrated regional health care system in Northern California. We also explored the association between COVID-19 outcomes and acute ( $<7$ days) vs chronic prednisone use, monotherapy vs combination therapy, specific medication subgroups (anti-TNF, thiopurine/DMARDS, JAK inhibitors), as well as underlying immune-mediated disease.

\section{METHODS}

\section{Study Design and Setting}

We conducted a retrospective cohort study among health plan members of Kaiser Permanente Northern California (KPNC), 
a large integrated health care delivery system that provides care for nearly 4.4 million members throughout the urban, suburban, and semirural regions in Northern California. The membership is diverse and similar in socioeconomic characteristics to the region's census demographics, including the proportions with commercial insurance, Medicare, and Medicaid. ${ }^{19}$

The Research Determination Committee for the Kaiser Permanente Northern California region determined the project does not meet the regulatory definition of research involving human subject per 45 CFR 46.102(d).

\section{Eligibility Criteria}

The study population consisted of health plan members aged 18 years or more with laboratory evidence of a SARS-CoV-2 diagnosis, defined as a positive PCR nasal swab between February 25 and September $9,2020 .^{20}$ The date of the positive SARS-CoV-2 PCR test served as the reference point for all exposure, outcome, and covariate measures.

\section{Data Sources}

Data for demographics, SARS-CoV-2 test results, medications, outcomes, and comorbidities were obtained from laboratory, pharmacy, medical visit, hospital, demographic, and membership electronic records.

\section{Medication Exposure}

The primary exposure was having any outpatient prescription fill for prednisone, immunomodulator, small-molecule, or biologic therapy in the 105 days prior to a positive SARS-CoV-2 PCR test. This window allowed the capture of prescriptions that may have been dispensed as 100-day supplies. We categorized non-steroid medications based on definitions and terms commonly understood across multiple autoimmune conditions. ${ }^{21}$ All medications were assessed using flag variables, allowing patients to be on any number of combination therapies.

Immune-modifying therapies selected were those used to treat either inflammatory bowel disease (ulcerative colitis, Crohn's disease), rheumatoid arthritis, spondyloarthropathies (ankylosing spondylitis, psoriatic arthritis), psoriasis, or systemic lupus erythematosus, or to prevent rejection from solid organ transplantation (Supplemental Table 1). The main oral steroid included was prednisone; there were few users of oral methylprednisolone, which we decided to remove for clear interpretation of prednisone results. Small-molecule therapies were grouped with biologic therapy to mitigate small cell size during multivariable analysis, similar to a prior study ${ }^{7}$. For therapies with counts less than 5, we presented and analyzed the data in larger aggregated groups or did not provide specific counts to protect personal health information (PHI).

\section{Outcome Ascertainment}

The primary outcome was severe illness after a SARS-CoV-2 diagnosis, defined as a composite outcome of hospitalization,
ICU admission, or death in the 45 days after a positive test or until September 10, 2020, whichever occurred first. Each outcome was also analyzed individually. Although we selected an outcome window of 45 days to allow sufficient time to capture all potentially related deaths to COVID- $19,{ }^{22}$ we repeated the analysis but restricted outcomes to 21 days, a common lag period between onset of disease and death. ${ }^{23}$

\section{Statistical Analysis}

Descriptive statistics were used to summarize demographic and clinical characteristics of cohort members. Categorical variables were expressed as proportions. Multivariable logistic regression analysis assessed the relationship between category of immune-modifying therapy before a positive SARS-CoV-2 PCR test and subsequent severe illness. Each component outcome (hospitalization, ICU admission, death) was also analyzed in separate models.

Models were adjusted for factors associated with severe illness from SARS-CoV-2, including age, gender, race/ethnicity, body mass index (BMI), and Charlson comorbidity index, as well as cancer, chronic obstructive pulmonary disease, diabetes, and hypertension. ${ }^{24-28}$ Models were also adjusted for underlying autoimmune disease as well as week of diagnosis, the latter to account for changing testing and treatment practices over time as well as the shortened length of followup for patients with a positive lab test near the end of the study period. For age, we selected the group aged 30-49 years as the reference group because it had the largest number of patients. BMI was categorized as underweight $\left(<18.5 \mathrm{~kg} / \mathrm{m}^{2}\right)$, normal weight $\left(18.5-24.9 \mathrm{~kg} / \mathrm{m}^{2}\right)$, overweight $\left(25.0-29.9 \mathrm{~kg} / \mathrm{m}^{2}\right)$, and obese $\left(\geq 30.0 \mathrm{~kg} / \mathrm{m}^{2}\right) .{ }^{29}$ Specific comorbidities (cancer, chronic obstructive pulmonary disease, diabetes, hypertension), as well as the Charlson comorbidity index, were assessed using a 1-year lookback period from date of SARS-CoV-2 testing. Specific immune conditions, including asthma (ICD10: J45); inflammatory bowel disease (K50-K51); solid organ transplantation of the kidney, heart, lung, or liver (Z94.0-Z94.4); psoriasis (L40 except L40.5); rheumatoid arthritis (M05M06); spondyloarthropathy (L40.5, M45); and systemic lupus erythematosus (M32), were assessed using a 5-year lookback period to ensure full capture of disease. To address potential concerns about collinearity between health conditions and medications, we repeated the analysis, but without adjusting for underlying autoimmune disease.

\section{Exploratory Analyses}

Exploratory analyses were performed to address additional data gaps, guided by clinical relevance, prior literature, and groupings with enough outcomes for meaningful analysis. ${ }^{7,9,10,13}$ These analyses assessed the relationship between severe SARS-CoV-2 and acute ( $<7$ days) vs longer ( $\geq 7$ days) steroid use, as well as combinations of medication classes and more granular groupings of therapies including TNF inhibitors, JAK inhibitors, and thiopurines/conventionaldisease-modifying 
antirheumatic drugs (DMARDs) (methotrexate, hydroxychloroquine, leflunomide). The remaining therapies were categorized as to whether they had an indication outside of autoimmune disease (typically transplant or cancer) or not (see Table 3 for a list). We did not assess outcomes based on steroid dose (1-9 mg vs. $10 \mathrm{mg}$ or more) ${ }^{7}$ as tapers were often written as free text elements that often fluctuated, and thus not accurately reflected in the electronic pharmacy record. Exploratory analyses were adjusted for the same factors as the primary analysis.

\section{RESULTS}

\section{Demographic Characteristics}

We identified 39,686 adults with a positive PCR nasal swab for SARS-CoV-2. A total of 2.4\% $(n=958)$ had received a prior prescription for oral prednisone, $0.8 \%(n=327)$ for an immunomodulator, $0.4 \%(n=152)$ for a biologic, and $0.1 \%(n$ = 47) for a small-molecule therapy (Table 1). These proportions were similar to the background population (data not shown). A total of 5069 had an underlying diagnosis of asthma, 1199 had an immune-mediated condition listed in Table 1, and 90 had undergone prior solid organ transplantation. A total of $10.0 \%(n=3977)$ had at least one outcome of interest. Most patients on an immunomodulator were prescribed either thiopurine, hydroxychloroquine, methotrexate, or leflunomide. Nearly half of the patients dispensed a small molecule were on a JAK inhibitor. Most patients on a biologic were on a TNF inhibitor, with less than $15 \%$ on rituximab. Additional characteristics of those with and without any severe COVID-19 outcome are shown in Table 1.

\section{Medication Use and Severe IIIness After SARS- CoV-2 Diagnosis}

After adjustment for covariates, outpatient prednisone use was associated with severe illness after diagnosis with SARSCoV-2 (odds ratio (OR) 1.31; 95\% confidence interval (CI) $1.08-1.60$ ), including hospitalization (OR 1.32; 95\% CI 1.081.60), ICU admission (OR 1.84; CI 1.38-2.46), and death (OR

Table 1 Characteristics of 39,686 Members Testing Positive for SARS-CoV-2 and a 45-Day Composite Outcome of any Hospitalization, ICU Admission, or Death

\begin{tabular}{|c|c|c|c|}
\hline Characteristic & $\begin{array}{l}\text { Any outcome } \\
N=3977 \\
N(\%)\end{array}$ & $\begin{array}{l}\text { No outcome } \\
N=35,709 \\
N(\%)\end{array}$ & $\begin{array}{l}\text { All patients } \\
N=39,686 \\
N(\%)\end{array}$ \\
\hline Female & $1833(46.1)$ & $18,835(52.8)$ & $20,668(52.1)$ \\
\hline \multicolumn{4}{|l|}{ Age (years) } \\
\hline $18-29$ & $245(6.2)$ & $9545(26.7)$ & $9790(24.7)$ \\
\hline $30-49$ & $998(25.1)$ & $15,747(44.1)$ & $16,745(42.2)$ \\
\hline $50-65$ & $1260(31.7)$ & $7874(22.1)$ & $9134(23.0)$ \\
\hline$>65$ & $1474(37.1)$ & $2543(7.1)$ & $4017(10.1)$ \\
\hline \multicolumn{4}{|l|}{ Race/ethnicity } \\
\hline Asian & $574(14.4)$ & $3970(11.1)$ & $4544(11.5)$ \\
\hline Black & $406(10.2)$ & $2408(6.7)$ & $2814(7.1)$ \\
\hline Hispanic & $1778(44.7)$ & $17,679(49.5)$ & $19,457(49.0)$ \\
\hline White & $898(22.6)$ & $7462(20.9)$ & $8360(21.1)$ \\
\hline Other/unknown & $321(8.1)$ & $4190(11.7)$ & 4511 (11.4) \\
\hline \multicolumn{4}{|l|}{ Body mass index } \\
\hline Underweight & $73(1.8)$ & $233(0.7)$ & $306(0.8)$ \\
\hline Healthy weight & $649(16.3)$ & $6133(17.2)$ & $6782(17.1)$ \\
\hline Overweight & $1021(25.7)$ & $10,478(29.3)$ & $11,499(29.0)$ \\
\hline Obese & $2052(51.6)$ & 14,869 (41.6) & $16,921(42.6)$ \\
\hline \multicolumn{4}{|l|}{ Charlson comorbidity score } \\
\hline 0 & $1691(42.5)$ & $27,220(76.2)$ & 28,911 (72.9) \\
\hline 1 & $699(17.6)$ & $5103(14.3)$ & $5802(14.6)$ \\
\hline$\geq 2$ & $1587(39.9)$ & $3386(9.5)$ & $4973(12.5)$ \\
\hline \multicolumn{4}{|l|}{ Specific comborbidities } \\
\hline Cancer & $164(4.1)$ & $406(1.1)$ & $570(1.4)$ \\
\hline Chronic obstructive pulmonary disease & $549(13.8)$ & $2068(5.8)$ & $2617(6.6)$ \\
\hline Diabetes & $1158(29.1)$ & $2055(5.8)$ & $3213(8.1)$ \\
\hline Hypertension & $1265(31.8)$ & $1535(4.3)$ & $2800(7.1)$ \\
\hline \multicolumn{4}{|l|}{ Specific immune conditions } \\
\hline Asthma & $745(18.7)$ & $4324(12.1)$ & $5069(12.8)$ \\
\hline Inflammatory bowel disease & $44(1.1)$ & $172(0.5)$ & $216(0.5)$ \\
\hline Organ transplantation & $39(1.0)$ & $51(0.1)$ & $90(0.2)$ \\
\hline Psoriasis & $98(2.5)$ & $453(1.3)$ & $551(1.4)$ \\
\hline Rheumatoid arthritis & $72(1.8)$ & $244(0.7)$ & $316(0.8)$ \\
\hline Spondyloarthropathy & $6(0.2)$ & $63(0.2)$ & $69(0.2)$ \\
\hline Systemic lupus erythematosis & $12(0.3)$ & $35(0.1)$ & $47(0.1)$ \\
\hline \multicolumn{4}{|l|}{ Therapies (not mutually exclusive) } \\
\hline Prednisone & $197(5.0)$ & $761(2.1)$ & $958(2.4)$ \\
\hline Conventional immunomodulator & $83(2.1)$ & $244(0.7)$ & $327(0.8)$ \\
\hline Biologic & $28(0.7)$ & $124(0.4)$ & $152(0.4)$ \\
\hline Small molecule & $9(0.2)$ & $38(0.1)$ & $47(0.1)$ \\
\hline None & $3723(93.6)$ & $34,683(97.1)$ & $38,406(96.8)$ \\
\hline
\end{tabular}


1.70; CI 1.17-2.47) (Table 2). Immunomodulator (OR 0.88; CI 0.57-1.34) and biologic/small-molecule therapy (OR 1.26; CI 0.79-2.00) were not associated.

\section{Sensitivity Analyses}

To address concerns about collinearity between health conditions and medications, we repeated the analysis, this time not adjusting for underlying autoimmune disease, but still adjusting for other covariates. For any outcome, the odds ratio for steroids was 1.38 (95\% CI 1.13-1.67), immunomodulators 1.05 (95\% CI 0.77-1.44), and biologic/small-molecule therapies 1.09 (95\% CI 0.71-1.68).

Restricting outcomes to 21 days after diagnosis did not meaningfully change the overall combined outcome for prednisone (OR 1.34, 95\% CI 1.10-1.63), immunomodulator (OR 0.94, 95\% CI 0.61-1.44), or small molecule/biologic (OR $1.29,95 \%$ CI 0.81-2.05), nor the individual outcomes, except that prednisone was no longer statistically significantly associated with death (OR 1.39, 95\% CI 0.88-2.18).

\section{Exploratory Analyses}

After adjustment for covariates, use of acute prednisone ( $<7$ days) was associated with ICU admission, but not other outcomes (Table 3). Chronic use ( $\geq 7$ days) had a comparable point estimate for death as in the main analysis but was no longer statistically significant (OR 1.59 ; 95\% CI 0.86-2.95). It remained significant for any outcome, inpatient admission, and ICU admission.

Neither monotherapy with immunomodulators (OR 0.76 ; 95\% CI 0.46-1.27) nor with biologics/small molecules (OR 0.82; 95\% CI 0.43-1.55) increased the odds of severe illness with SARS-CoV-2, whereas prednisone monotherapy did increase the odds (OR 1.25; 95\% CI 1.01-1.54). Patients on multiple therapies had increased odds of severe illness, but only the combination of a biologic/small molecule with prednisone was statistically significant (OR 3.57; 95\% CI 1.17-10.85) (Table 3).

Use of thiopurines/traditional DMARDs prior to diagnosis of SARS-CoV-2 had a reduced odds of severe illness (OR 0.53; 95\% CI 0.32-0.88), JAK inhibitors had an increased odds (OR 3.35, 95\% CI 1.16-9.67), and anti-TNF therapy was not associated (OR 0.45, 95\% CI 0.18-1.08) (Table 3). Among the remaining therapies, prior use of biologic/small-molecule therapies used exclusively in autoimmune diseases (see list in Table 3) did not have an increased odds of severe illness (OR $0.83 ; 95 \%$ CI $0.37-1.89)$. In contrast, biologics and immunomodulators with an indication beyond autoimmune disease (i.e., typically cancer or transplant) had an increased odds (OR 3.84, 95\% CI 2.01-7.35).

Table 2 Adjusted Associations of 45-Day Severe Outcomes of Patients Testing Positive for SARS-CoV-2. $N=39,686$

\begin{tabular}{|c|c|c|c|c|}
\hline & $\begin{array}{l}\text { Any outcome } \\
\text { OR }(95 \% \text { CI) } \\
N=3977\end{array}$ & $\begin{array}{l}\text { Hospitalization } \\
\text { OR }(95 \% \text { CI }) \\
N=3819\end{array}$ & $\begin{array}{l}\text { ICU admission } \\
\text { OR (95\% CI) } \\
N=991\end{array}$ & $\begin{array}{l}\text { Death } \\
\text { OR }(95 \% \text { CI) } \\
N=600\end{array}$ \\
\hline \multicolumn{5}{|l|}{ Medication class (vs none) } \\
\hline Prednisone & $1.31(1.08-1.60)$ & $1.32(1.08-1.60)$ & $1.84(1.38-2.46)$ & $1.70(1.17-2.47)$ \\
\hline Conventional immunomodulator & $0.88(0.57-1.34)$ & $0.99(0.65-1.51)$ & $0.95(0.49-1.85)$ & $1.71(0.85-3.47)$ \\
\hline Small molecule or biologic & $1.26(0.79-2.00)$ & $1.27(0.80-2.01)$ & $1.32(0.64-2.69)$ & $1.51(0.66-3.48)$ \\
\hline \multicolumn{5}{|l|}{ Immune condition (vs none) } \\
\hline Asthma & $1.08(0.98-1.20)$ & $1.11(1.00-1.23)$ & $1.10(0.92-1.32)$ & $0.85(0.67-1.07)$ \\
\hline Inflammatory bowel disease & $1.22(0.82-1.81)$ & $1.15(0.77-1.72)$ & $0.83(0.41-1.69)$ & $0.76(0.35-1.68)$ \\
\hline Organ transplantation & $1.84(1.00-3.40)$ & $1.86(1.01-3.40)$ & $1.58(0.67-3.71)$ & $0.69(0.24-1.95)$ \\
\hline Psoriasis & $1.15(0.89-1.49)$ & $1.17(0.90-1.51)$ & $1.63(1.10-2.39)$ & $0.92(0.53-1.58)$ \\
\hline Spondyloarthopathy & $0.37(0.14-0.97)$ & $0.38(0.15-0.99)$ & $0.19(0.02-1.51)$ & $0.37(0.04-3.08)$ \\
\hline Rheumatoid arthritis & $0.95(0.66-1.36)$ & $0.93(0.65-1.34)$ & $0.97(0.55-1.69)$ & $0.67(0.36-1.24)$ \\
\hline Systemic lupus erythematosis & $1.22(0.53-2.83)$ & $1.31(0.57-2.99)$ & $1.11(0.32-3.80)$ & $1.00(0.25-4.00)$ \\
\hline Charlson comorbidity score & $1.15(1.13-1.18)$ & $1.12(1.09-1.14)$ & $1.07(1.04-1.11)$ & $1.18(1.14-1.23)$ \\
\hline Hypertension & $3.19(2.88-3.53)$ & $3.28(2.95-3.63)$ & $3.31(2.80-3.91)$ & $2.24(1.83-2.73)$ \\
\hline Female (vs. male) & $0.68(0.63-0.73)$ & $0.67(0.63-0.73)$ & $0.46(0.40-0.53)$ & $0.63(0.53-0.75)$ \\
\hline \multicolumn{5}{|l|}{ Age, years } \\
\hline $18-29$ & $0.48(0.42-0.56)$ & $0.48(0.42-0.56)$ & $0.41(0.29-0.58)$ & $0.31(0.11-0.89)$ \\
\hline $30-49$ & 1.00 (ref) & 1.00 (ref) & 1.00 (ref) & 1.00 (ref) \\
\hline $50-65$ & $2.00(1.83-2.20)$ & $2.02(1.84-2.22)$ & $2.55(2.13-3.06)$ & $7.03(4.52-10.94)$ \\
\hline$>65$ & $4.87(4.35-5.45)$ & $4.52(4.03-5.06)$ & $4.66(3.77-5.75)$ & $32.91(21.30-50.84)$ \\
\hline \multicolumn{5}{|l|}{ Race/ethnicity } \\
\hline Asian & $1.74(1.53-1.98)$ & $1.82(1.60-2.07)$ & $2.07(1.63-2.63)$ & $0.82(0.61-1.10)$ \\
\hline Black & $1.55(1.34-1.80)$ & $1.60(1.38-1.86)$ & $1.84(1.41-2.39)$ & $1.03(0.76-1.40)$ \\
\hline Hispanic & $1.43(1.29-1.58)$ & $1.49(1.35-1.64)$ & $2.02(1.68-2.44)$ & $0.89(0.71-1.11)$ \\
\hline White & 1.00 (ref) & 1.00 (ref) & 1.00 (ref) & 1.00 (ref) \\
\hline & $1.18(1.01-1.37)$ & $1.18(1.01-1.38)$ & $1.55(1.16-2.06)$ & $0.99(0.70-1.39)$ \\
\hline \multicolumn{5}{|l|}{ Body mass index } \\
\hline Underweight & $2.20(1.57-3.10)$ & $1.47(1.02-2.12)$ & $1.31(0.71-2.45)$ & $3.13(2.00-4.92)$ \\
\hline Normal weight & 1.00 (ref) & 1.00 (ref) & 1.00 (ref) & 1.00 (ref) \\
\hline Overweight & $0.97(0.86-1.09)$ & $1.03(0.92-1.17)$ & $1.06(0.86-1.32)$ & $0.55(0.43-0.70)$ \\
\hline Obese & $1.60(1.43-1.79)$ & $1.72(1.53-1.92)$ & $1.66(1.35-2.04)$ & $0.81(0.65-1.02)$ \\
\hline
\end{tabular}

All models also adjust for week of SARS-CoV-2-positive lab test

*Also adjusts for missing/unknown status 
Table 3 Exploratory Analyses of Specific Medication Exposures and Any Severe COVID-19 Outcome

\begin{tabular}{|c|c|c|}
\hline & $\begin{array}{l}\text { Number of outcomes/number } \\
\text { of cases }\end{array}$ & $\begin{array}{l}\text { Adjusted } \\
\text { OR (95\% CI) } \\
N=3977\end{array}$ \\
\hline \multicolumn{3}{|l|}{ Original analysis (Table2) } \\
\hline No immune-mediated medication & $3723 / 38,406$ & Ref \\
\hline Prednisone & $197 / 958$ & $1.31(1.08-1.60)$ \\
\hline Immunomodulator & $83 / 327$ & $0.88(0.57-1.34)$ \\
\hline Small-molecule/biologic therapy & $36 / 193$ & $1.26(0.79-2.00)$ \\
\hline \multicolumn{3}{|l|}{ Short-term vs longer prednisone ${ }^{*}$} \\
\hline No immune-mediated medication & $3723 / 38,406$ & Ref \\
\hline Short-term prednisone $(<7$ days $)$ & $62 / 432$ & $1.08(0.80-1.48)$ \\
\hline Longer prednisone $(\geq 7$ days) & $123 / 491$ & $1.49(1.15-1.93)$ \\
\hline Immunomodulator & $83 / 327$ & $0.85(0.56-1.31)$ \\
\hline Small-molecule/biologic therapy & $36 / 193$ & $1.25(0.78-1.98)$ \\
\hline \multicolumn{3}{|l|}{ Monotherapy vs combination therapy } \\
\hline No immune-mediated medication & $3723 / 38,406$ & Ref \\
\hline Monotherapy & & \\
\hline Prednisone & $149 / 821$ & $1.25(1.01-1.54)$ \\
\hline Immunomodulator & $34 / 167$ & $0.76(0.46-1.27)$ \\
\hline Small molecule/biologic & $16 / 115$ & $0.82(0.43-1.55)$ \\
\hline \multicolumn{3}{|l|}{ Combination therapy } \\
\hline Prednisone + immunomodulator & $35 / 99$ & $1.12(0.56-2.25)$ \\
\hline Prednisone + small molecule/biologic & $6 / 17$ & $3.57(1.17-10.85)$ \\
\hline Immunomodulator + small molecule/biologic & $7 / 40$ & $1.17(0.45-3.04)$ \\
\hline All three & $7 / 21$ & $2.99(0.94-9.49)$ \\
\hline \multicolumn{3}{|l|}{ Selected medication groupings } \\
\hline No immune-mediated medication & $3723 / 38,406$ & Ref \\
\hline Prednisone & $197 / 958$ & $1.27(1.04-1.55)$ \\
\hline Thiopurines/traditional DMARDs ${ }^{\dagger}$ & $34 / 220$ & $0.53(0.32-0.88)$ \\
\hline Anti-TNF & $7 / 94$ & $0.45(0.18-1.08)$ \\
\hline JAK-I & $7 / 23$ & $3.35(1.16-9.67)$ \\
\hline Other therapies used only in immune-mediated inflammatory disease ${ }^{i \dagger}$ & $9 / 66$ & $0.83(0.37-1.89)$ \\
\hline Other therapies used also in other diseases (i.e., transplant, cancer) ${ }^{\S}$ & $60 / 129$ & $3.84(2.01-7.35)$ \\
\hline
\end{tabular}

All models adjust for patient age, sex, racelethnicity, body mass index, immune-mediated disease (asthma, inflammatory bowel disease, organ transplantation, psoriasis, rheumatoid arthritis, spondyloarthropathy, lupus), hypertension, Charlson comorbidity score, and week of SARS-CoV-2positive lab test. Therapies with at least 1 patient are listed

*35 patients missing length of prednisone

tAzathioprine, 6-mercaptopurine, methotrexate, hydroxychloroquine, leflunomide

${ }^{+}$Apremilast, tocilizumab, ustekinumab, guselkumab, secukinumab, vedolizumab, belimumab, abatacept

${ }^{\S}$ Cyclosporine, tacrolimus, everolimus, cyclophosphamide, mycophenolate, rituximab

\section{Immune-Mediated Disease}

Organ transplantation was associated with a borderline increased odds of severe illness with SARS-CoV-2 (OR 1.84, 95\% CI 1.00-3.40) while spondyloarthropathy had a reduced odds (OR $0.37,95 \%$ CI $0.14-0.97$ ) (Table 2). Transplantation increased the odds of hospitalization, as did asthma (borderline) while spondyloarthropathy had reduced odds. Psoriasis was associated with increased odds of ICU admission. None of the other immune conditions listed in Table 2 were associated with increased odds of ICU admission or mortality.

\section{Other Prognostic Factors}

A higher Charlson score, hypertension, male sex, age $>50$, non-white race, and extremes of BMI (underweight and obese) were all associated with severe illness after diagnosis with SARS-CoV-2(Table 2).

\section{DISCUSSION}

In this study, we addressed whether the use of corticosteroids and other immune-weakening medicines prior to a diagnosis of SARS-CoV-2 increased the risk of subsequent hospitalization, ICU admission, and death. The present study builds on the emerging but limited data on this topic derived primarily from international registries, referral center studies, and administrative claims data. ${ }^{6-17} \mathrm{We}$ found outpatient use of prednisone was associated individually and in aggregate with hospitalization, ICU admission, and death, indicating a strong effect across the spectrum of severity. In contrast, immunomodulator and biologic/small-molecule therapy were not associated.

The association between steroid use prior to the SARSCoV-2 diagnosis and subsequent hospitalization, ICU admission, and death observed in our study is similar to what has been observed in single-specialty rheumatology, IBD, and psoriasis studies. ${ }^{6-10,12,15}$ This finding is also consistent with the known effects of steroids, which worsen viremia, prolong the viral shedding time, and increase the severity of infection by diminishing the immune system's antiviral defenses. ${ }^{30}$ While we were unable to provide a more granular dosedependent exposure with outcomes, the consistency in results for prednisone (except for death), when use was assessed as $\geq$ 7 days and outcomes were restricted to 21 days, supports a biologic association between chronic outpatient prednisone 
and worse outcomes after SARS-CoV-2 diagnosis, although residual confounding cannot be completely excluded.

In contrast, immunomodulators and biologic/smallmolecule therapies prior to SARS-CoV-2 diagnosis were not associated with severe illness in the primary analysis, consistent with results from other retrospective studies. ${ }^{6-8,10,16,18}$ These therapies can also affect the immune system, and thus, the contrast with prednisone is notable. The difference in point estimates for these therapies when used as monotherapy compared to combination therapy supports current recommendations that chronic use of immunomodulator, biologic, and small-molecule therapy does not appear to meaningfully increase the risk of severe outcomes after diagnosis of SARSCoV-2. Our results are consistent with recommendations that patients should not change or discontinue therapy for fear of worse outcomes. ${ }^{31-33}$ Of course, appropriate measures should be taken to avoid infection and mitigate severe infection.

Our exploratory analyses are consistent with other singlespecialty studies reporting anti-TNF therapy does not increase the odds of severe illness with SARS-CoV-2. ${ }^{6,8,10} \mathrm{TNF}$ is significantly elevated in severe COVID-19, and its inhibition could provide benefit in reducing cytokine storm, a systemic inflammatory response to SARS-CoV-2 believed to contribute to its morbidity and mortality. ${ }^{7}$ Our observed reduced odds of severe illness with thiopurines/DMARDs is similar to the few other available studies, which have shown no increased risk with methotrexate or hydroxychloroquine, ${ }^{9,10}$ except for a referral study showing borderline association with hydroxychloroquine (OR 4.79; 95\% CI 1.00-22.86). ${ }^{10}$ Data are sparse for other therapies. Two registry and one referral study have individually reported no association with severe COVID-19 for CTLA4-Ig, ${ }^{9}$ anti-IL6, ${ }^{9}$ anti-IL17, ${ }^{9,10}$ anti-IL12/23, ${ }^{13}$ and anti-integrin therapy, ${ }^{13}$ with sparse data for other anti-cytokine therapies. We caution that ongoing accrual of data is needed to better assess the risk of severe outcomes with individual therapies.

We did find prior use of therapies with a strong use outside of autoimmune disease (typically transplant or cancer) also increased the odds of severe illness. A registry study reported similar findings for mycophenolate mofetil (OR 7.67; 95\% CI 1.73-28.04) as well as B cell-depleting rituximab (OR 4.34; 95\% CI 1.77-10.63), ${ }^{9}$ which are used in autoimmune disease but also transplant and cancer, respectively. Data for other therapies were not reported. ${ }^{9}$ The reasons for this observation may be several, including more profound immunosuppression compared to other therapies, indication bias, or unmeasured confounders, such as frailty in patients on these therapies. ${ }^{34}$

It is notable our retrospective study showed outpatient exposure to prednisone and JAK inhibitors prior to SARSCoV-2 diagnosis had an increased odds of severe SARSCOV-2 outcomes whereas randomized trials of intravenous corticosteroids or tofacitinib (JAK inhibitor) used in hospitalized patients already infected with SARS-CoV-2 and requiring respiratory support reduced mortality ${ }^{35,36}$ (presumably from mitigating the cytokine storm). ${ }^{37} \mathrm{~A}$ trend toward increased risk of severe COVID-19 with JAK-inhibitor therapy prior to SARS-CoV-2 diagnosis has been observed in a retrospective French registry and a New York referral center study, but not on univariate analysis in an IBD registry study. ${ }^{9,10,14}$ All three studies reported low patient numbers and concerns for confounding. Thus, while it may be that the aggravating, beneficial, or neutral effect of outpatient immunosuppressive therapy on COVID-19 outcomes may depend on timing and the balance of antiviral containment and anti-inflammatory response, ${ }^{9,30}$ this hypothesis should be considered speculative in the absence of more definitive data.

Although not the primary aim of study, this study confirmed the relationship between older age, male gender, hypertension, preexisting comorbidities, non-white race, and obesity with severe illness with SARS-CoV-2. ${ }^{24-27,38}$ It also provided novel data in that most immune-mediated inflammatory diseases in our study did not increase the odds for ICU admission or mortality, after adjusting for immunosuppressive medication type, specific comorbidities, and demographic risk factors. We found organ transplantation increased the odds of hospitalization and asthma had a borderline association, whereas psoriasis increased the odds of ICU admission, the latter perhaps due to its strong association with significant comorbid conditions. ${ }^{39}$

There are several study limitations. We grouped therapies into clinically meaningfully and commonly used categories, but acknowledge that these treatments have different mechanisms of action, different effects on the immune system, and potentially different effects on outcomes depending on when used in the natural history of SARS-CoV-2 infection. ${ }^{40}$ Ongoing studies are necessary to accrue sufficient data to provide risk estimates for individual therapies. It is also possible that outcomes occurring outside of the health setting and not captured in membership files may have been missed, although this is uncommon. Finally, disease severity is not well captured in administrative data. While prednisone could be a marker of more severe immune-mediated disease or other condition requiring hospitalization within 45 days, we feel it should not be associated with short-term ICU admission and death if a confounder, particularly after adjustment for age, disease, and comorbidity. Thus, while we propose a true biologic association between prednisone and SARS-CoV-2 infection leading to the observed outcomes, residual confounding cannot be excluded completely.

A key strength of the study is the setting, conducted within a single, large, integrated, community-based health care system. This permitted inclusion of a racially and medically diverse population of patients with detailed collection of medication, outcome, and key prognostic factors. This increased the generalizability of the findings as well as permitted overcoming potential issues with prior studies, including small sample size, limited inclusion of key variables for multivariable analysis, convenience sampling of physician-reported cases, and finally pooling of data from many different countries, each with their own health system, practices, and resources. 
In summary, in a large, diverse, community-based population, outpatient use of prednisone was associated with severe illness after diagnosis of SARS-CoV-2. Immunomodulator and biologic/small-molecule therapies were not associated. We propose our findings can help risk-benefit discussions in the clinic and support recommendations that patients should not stop or reduce immunomodulator, biologic, or smallmolecule therapy due to concerns of an elevated risk of severe illness from SARS-CoV-2. ${ }^{2-4,31-33}$ For those therapies where we showed an increased odds, providers can discuss with patients the limitations of the current data and review evolving medical society recommendations regarding medication management, ${ }^{31-33}$ as well as reinforce strategies to mitigate risk of severe SARS-CoV-2 infection, which currently involves vaccination, particularly in vulnerable groups.

Corresponding Author: Fernando S. Velayos, M.D. M.P.H.; Division of Gastroenterology and Hepatology, Kaiser Permanente San Francisco Medical Center, San Francisco, CA, USA (e-mail: Fernando. Velayos@kp.org).

Supplementary Information The online version contains supplementary material available at https://doi.org/10.1007/s11606-02107152-2.

\section{Declarations:}

Conflict of Interest: The authors declare that they do not have a conflict of interest.

\section{REFERENCES}

1. Harpaz R, Dahl RM, Dooling KL. Prevalence of Immunosuppression Among US Adults, 2013. JAMA. 2016;316(23):2547-2548.

2. Goodsall TM, Han S, Bryant RV. Understanding attitudes, concerns, and health behaviors of patients with inflammatory bowel disease during the coronavirus disease 2019 pandemic. J Gastroenterol Hepatol. 2020.

3. Mehta B, Jannat-Khah D, Mancuso CA, et al. Geographical variations in COVID-19 perceptions and patient management: a national survey of rheumatologists. Semin Arthritis Rheum. 2020;50(5): 1049-1054.

4. Agrawal M, Brenner EJ, Zhang X, Colombel JF, Kappelman MD, Ungaro RC. Physician practice patterns on holding inflammatory bowel disease medications due to COVID-19 in the SECURE-IBD registry. $J$ Crohns Colitis. 2020.

5. Certain Medical Conditions and Risk for Severe COVID-19 illness. https://www.cdc.gov/coronavirus/2019-ncov/need-extra-precautions/ people-with-medical-conditions.html\#immunocompromised-state. Accessed 2/22/2021.

6. Brenner EJ, Ungaro RC, Gearry RB, et al. Corticosteroids, But Not TNF Antagonists, Are Associated With Adverse COVID-19 Outcomes in Patients With Inflammatory Bowel Diseases: Results From an International Registry. Gastroenterology. 2020;159(2):481-491 e483.

7. Gianfrancesco $\mathbf{M}$, Hyrich KL, Al-Adely S, et al. Characteristics associated with hospitalisation for COVID-19 in people with rheumatic disease: data from the COVID-19 Global Rheumatology Alliance physician-reported registry. Ann Rheum Dis. 2020;79(7):859-866.

8. Bezzio C, Saibeni S, Variola A, et al. Outcomes of COVID-19 in 79 patients with IBD in Italy: an IG-IBD study. Gut. 2020;69(7):1213-1217.

9. consortium FRSSSCI, contributors. Severity of COVID-19 and survival in patients with rheumatic and inflammatory diseases: data from the French RMD COVID- 19 cohort of 694 patients. Ann Rheum Dis. 2020.

10. Haberman RH, Castillo R, Chen A, et al.COVID-19 in Patients With Inflammatory Arthritis: A Prospective Study on the Effects of
Comorbidities and Disease-Modifying Antirheumatic Drugs on Clinical Outcomes. Arthritis Rheumatol. 2020;72(12):1981-1989.

11. Lukin DJ, Kumar A, Hajifathalian K, et al. Baseline Disease Activity and Steroid Therapy Stratify Risk of COVID-19 in Patients With Inflammatory Bowel Disease. Gastroenterology. 2020;159(4): 1541-1544 e1542.

12. Singh S, Khan A, Chowdhry M, Bilal M, Kochhar GS, Clarke K. Risk of Severe Coronavirus Disease 2019 in Patients With Inflammatory Bowel Disease in the United States: A Multicenter Research Network Study. Gastroenterology. 2020;159(4): 1575-1578 e1574.

13. Ungaro RC, Brenner EJ, Gearry RB, et al. Effect of IBD medications on COVID-19 outcomes: results from an international registry. Gut. 2020.

14. Agrawal M, Brenner EJ, Zhang $\mathbf{X}$, et al. Characteristics and Outcomes of IBD Patients with COVID-19 on Tofacitinib Therapy in the SECUREIBD Registry. Inflamm Bowel Dis. 2020.

15. Mahil SK, Dand N, Mason KJ, et al. Factors associated with adverse COVID-19 outcomes in patients with psoriasis-insights from a global registry-based study. J Allergy Clin Immunol. 2020.

16. Burke KE, Kochar B, Allegretti JR, et al. Immunosuppressive Therapy and Risk of COVID-19 Infection in Patients With Inflammatory Bowel Diseases. Inflamm Bowel Dis. 2020.

17. Damiani G, Pacifico A, Bragazzi NL, Malagoli P. Biologics increase the risk of SARS-CoV-2 infection and hospitalization, but not ICU admission and death: Real-life data from a large cohort during red-zone declaration. Dermatol Ther. 2020;33(5):e13475.

18. Andersen KM, Mehta HB, Palamuttam N, et al. Association Between Chronic Use of Immunosuppresive Drugs and Clinical Outcomes From Coronavirus Disease 2019 (COVID-19) Hospitalization: A Retrospective Cohort Study in a Large US Health System. Clin Infect Dis. 2021.

19. Gordon N, Lin T. The Kaiser Permanente Northern California Adult Member Health Survey. Perm J. 2016;20(4):15-225.

20. Smith D, Anderson C. First U.S. coronavirus case of unknown origin confirmed in Northern California, CDC says https://www.sacbee.com/ news/california/article240674471.html. Accessed 2/22/2021.

21. Smolen JS, van der Heijde D, Machold KP, Aletaha D, Landewe R. Proposal for a new nomenclature of disease-modifying antirheumatic drugs. Ann Rheum Dis. 2014;73(1):3-5.

22. Chen Y, Li T, Ye Y, Chen Y, Pan J. Impact of Fundamental Diseases on Patients With COVID-19. Disaster Med Public Health Prep. 2020; 14(6):776-781

23. Yao T, Gao Y, Cui Q, et al. Clinical characteristics of a group of deaths with COVID-19 pneumonia in Wuhan, China: a retrospective case series. BMC Infect Dis. 2020;20(1):695.

24. Price-Haywood EG, Burton J, Fort D, Seoane L. Hospitalization and Mortality among Black Patients and White Patients with Covid-19. N Engl J Med. 2020;382(26):2534-2543.

25. Tuty Kuswardhani RA, Henrina J, Pranata R, Anthonius Lim M, Lawrensia S, Suastika $\mathbf{K}$. Charlson comorbidity index and a composite of poor outcomes in COVID-19 patients: A systematic review and metaanalysis. Diabetes Metab Syndr. 2020;14(6):2103-2109.

26. Scully EP, Haverfield J, Ursin RL, Tannenbaum C, Klein SL. Considering how biological sex impacts immune responses and COVID19 outcomes. Nat Rev Immunol. 2020;20(7):442-447.

27. Reilev M, Kristensen KB, Pottegard A, et al. Characteristics and predictors of hospitalization and death in the first 11122 cases with a positive RT-PCR test for SARS-CoV-2 in Denmark: a nationwide cohort. Int $J$ Epidemiol. 2020;49(5): 1468-1481.

28. Guan H, Sundararajan V, Halfon P, et al. Coding algorithms for defining comorbidities in ICD-9-CM and ICD-10 administrative data. Med Care. 2005;43(11):1130-1139.

29. Clinical guidelines on the identification, evaluation, and treatment of overweight and obesity in adults: executive summary. Expert Panel on the Identification, Evaluation, and Treatment of Overweight in Adults. Am $J$ Clin Nutr. 1998;68(4):899-917.

30. Robinson PC, Morand E. Divergent effects of acute versus chronic glucocorticoids in COVID-19. Lancet Rheumatol. 2021;3(3):e168-e170.

31. Rubin DT, Feuerstein JD, Wang AY, Cohen RD. AGA Clinical Practice Update on Management of Inflammatory Bowel Disease During the COVID-19 Pandemic: Expert Commentary. Gastroenterology. 2020;159(1):350-357.

32. Mikuls TR, Johnson SR, Fraenkel L, et al. American College of Rheumatology Guidance for the Management of Rheumatic Disease in Adult Patients During the COVID-19 Pandemic: Version 3. Arthritis Rheumatol. 2020. 
33. Guidance on the Use of Immunosuppressive Agents (American Academy of Dermatology). https://www.aad.org/member/practice/coronavirus / clinical-guidance/biologics. Accessed 2/22/2021.

34. Hewitt J, Carter B, Vilches-Moraga A, et al. The effect of frailty on survival in patients with COVID-19 (COPE): a multicentre, European, observational cohort study. Lancet Public Health. 2020;5(8):e444-e451.

35. Group WHOREAfC-TW, Sterne JAC, Murthy S, et al. Association Between Administration of Systemic Corticosteroids and Mortality Among Critically Ill Patients With COVID-19: A Meta-analysis. JAMA. 2020;324(13):1330-1341

36. Guimaraes PO, Guirk D, Furtado RH, et al. Tofacitinib in Patients Hospitalized with Covid-19 Pneumonia. N Engl J Med. 2021;385(5):406415.

37. Fajgenbaum DC, June CH. Cytokine Storm. $N$ Engl $J$ Med. 2020;383(23):2255-2273.
38. Nguyen GC, Gulamhusein A, Bernstein CN. 5-aminosalicylic acid is not protective against colorectal cancer in inflammatory bowel disease: a meta-analysis of non-referral populations. Am $J$ Gastroenterol. 2012;107(9):1298-1304; quiz 1297, 1305.

39. Yeung H, Takeshita J, Mehta NN, et al. Psoriasis severity and the prevalence of major medical comorbidity: a population-based study. JAMA Dermatol. 2013;149(10):1173-1179.

40. Catanzaro M, Fagiani F, Racchi M, Corsini E, Govoni S, Lanni C. Immune response in COVID-19: addressing a pharmacological challenge by targeting pathways triggered by SARS-CoV-2. Signal Transduct Target Ther. 2020;5(1):84.

Publisher's Note: Springer Nature remains neutral with regard to jurisdictional claims in published maps and institutional affiliations. 